\title{
L'efectivitat de les tècniques de relaxació en la malaltia del Parkinson Revisió bibliogràfica
}

\author{
Teresa Balaguer Pallarés \\ al316128@uji.es \\ Inés Balaguer Pallarés \\ al228012@uji.es \\ Lledó Guillamón Gimeno \\ guillamm@uji.es
}

\section{Resum}

Introducció: La malaltia de Parkinson és un trastorn neurodegeneratiu originat per la degeneració de les neurones dopaminèrgiques de la substància nigra del cervell, àrea encarregada de la coordinació de l'activitat, el to muscular i els moviments. Es contemplen les tècniques de relaxació dintre del tractament no farmacològic adreçat als símptomes motors de la malaltia, que interfereixen progressivament en la realització de les activitats de la vida diària.

L'objectiu d'aquest estudi és conèixer si les tècniques de relaxació són efectives per millorar els símptomes motos de la malaltia de Parkinson.

Metodologia: Es va realitzar una recerca bibliogràfica amb els descriptors en llenguatge controlat i natural «Enfermedad de Parkinson» i «Técnicas de relajación» en les bases de dades Pubmed-Medline, Pubmed-Clinical Queries, Biblioteca Cochrane Plus i Biblioteca Virtual en Salut durant el mes de març de 2017. S'utilitzaren filtres temporals (2010-2017) i idiomàtics (anglès i espanyol).

Resultats: S'obtingueren un total de $\mathrm{N}=101$ articles: $77,23 \%$ en PubmedMedline, 7,92 \% en Pubmed-Clinical Queries, 10,89\% en Biblioteca Cochrane Plus i $3,96 \%$ en BVS; de la mostra resultant es van haver de rebutjar $88,12 \%$ $(n=89)$ per no guardar relació amb l'objectiu de la recerca i un $4,95 \%$ per repetició del mateix article en les diferents bases de dades.

Conclusions: $\mathrm{Hi}$ ha escassa literatura sobre l'efectivitat de les tècniques de relaxació sobre els símptomes motors en la malaltia de Parkinson. Les publicacions disponibles fan referència a les tècniques de relaxació com a complement del tractament farmacològic. Les tècniques de relaxació són efectives quan es comparen amb la música o la fisioteràpia. En canvi, al comparar-les amb l'exercici físic aeròbic, aquest últim dóna millors resultats, ja que millora els símptomes motors i, a més, també hi ha una millora en la marxa i la mobilitat del malalt.

Paraules clau: malaltia de Parkinson, tècniques de relaxació, ansietat, símptomes motors, efectivitat. 


\begin{abstract}
Introduction: Parkinson's disease is a neurodegenerative disorder caused by degeneration of dopaminergic neuron nigra brain, an area responsible for the coordination of activity, muscle tone and movements. Relaxation techniques are contemplated within the non-pharmacological treatment aimed at the motive symptoms of the disease, which progressively interfere with the realization of daily life activities.

The objective of this study is to know if relaxation techniques are effective in improving the motorbike symptoms of Parkinson's disease.

Methodology: A bibliographic search was carried out with the descriptors in controlled and natural language "Parkinson's Disease" and "Relaxation techniques" in the Pubmed-Medline, Pubmed-Clinical Queries databases, the Cochrane Plus Library and the Virtual Health Library during The month of March 2017. Temporary filters (2010-2017) and idiomatic (English and Spanish) were used.

Results: A total of $\mathrm{N}=101$ articles were obtained: $77.23 \%$ in Pubmed-Medline, $7.92 \%$ in Pubmed-Clinical Queries, $10.89 \%$ in the Cochrane Plus Library and $3.96 \%$ in BVS; Of the resulting sample, $88.9 \%(n=89)$ had to be rejected because they were not related to the purpose of the search and $4.95 \%$ were rejected because the same article was found in the different databases.

Conclusions: There is little literature about the effectiveness of relaxation techniques on motor symptoms in Parkinson's disease. The available publications refer to relaxation techniques as a complement to the pharmacological treatment. Relaxation techniques are effective when compared to music or physiotherapy. On the other hand, when compared to aerobic physical exercise, the latter gives better results, as it improves the motor symptoms and, in addition, there is also an improvement in the movement and mobility of the patient.
\end{abstract}

Keywords: Parkinson's disease, relaxation techniques, anxiety, motor symptoms, effectiveness.

\title{
Introducció
}

La malaltia de Parkinson (MP), definida per la Federació Espanyola de Parkinson, és una malaltia que afecta el sistema nerviós en l'àrea encarregada de la coordinació de l'activitat, el to muscular i els moviments.

Fou descrita per primera vegada l'any 1817 pel metge anglès James Parkinson (Federació Espanyola de Parkinson). Aquesta malaltia és un trastorn crònic neurodegeneratiu del moviment progressiu, s'origina amb la degeneració o la mort progressiva de les neurones dopaminèrgiques de la substància nigra del cervell. Aquestes neurones segreguen la dopamina, un neurotransmissor primari que transmet els senyals des de la substància nigra a altres àrees del cervell $i$, a través d'impulsos químics i elèctrics, permet el funcionament coordinat dels músculs i el moviment del cos (Nausieda, Bock i Dowling 2002).

La neuròloga Luquín Pulido de la Clínica Universitària de Navarra defineix la síndrome parkinsoniana com el fruit d'una disfunció dels ganglis basals, els quals estan formats per l'estriat (nucli caudat i putamen), la substància nigra (pars compacta i reticulata), globus pàl-lid medial, nucli subtalàmic i nuclis ventral anterior i lateral del tàlem. La degeneració de les neurones dopaminèrgiques que projecten a l'estriat produeix una disminució de dopamina en l'estriat i açò provoca l'alteració del processament de la 
informació que s'origina en el còrtex cerebral; el resultat d'aquesta anomalia endògena és l'alteració del control del moviment (Luquín Pulido 2012).

Els quatre principals símptomes motors de la MP són: tremolor, bradicinèsia, rigidesa d'extremitats i de tronc i inestabilitat postural. Aquests es manifesten quan ja s'ha produït una pèrdua important de les neurones esmentades i com a conseqüència, una disminució dels nivells de dopamina (Federació Espanyola de Parkinson sense data; Nausieda, Bock i Dowling 2002).

La prevalença d'aquesta malaltia es relaciona directament amb l'edat, sent en els majors de 70 anys més de 500/100 000 habitants. La incidència anual per a aquesta malaltia varia entre 4,9 i 26/100 000 habitants i el pic d'incidència ocorre entre els 6069 anys d'edat (Luquín Pulido 2012).

La MP es classifica en estadis clínics depenent del grau d'afectació, sent l'estadi 0 sense afectació i l'estadi 5 el d'afectació més severa. El diagnòstic d'aquesta malaltia sempre és neuropatològic i es basa en la identificació en el cervell dels marcadors: pèrdua neuronal dopaminèrgica $i$ cossos de Lewy. En els primers estadis resulta complex un diagnòstic amb exactitud (Nausieda, Bock i Dowling 2002; Luquín Pulido 2012).

Els símptomes motors d'aquesta malaltia afecten directament l'estat físic de la persona i, a mesura que avança la malaltia, interfereixen cada vegada més en la realització de les activitats de la vida diària (AVD). Aquesta situació pot abocar el pacient a un estat d'ansietat que accentua els esmentats símptomes. El tractament farmacològic juga un paper important en el control motor d'aquests tipus de pacients, però entenent la relaxació com un estat de consciència, de calma i d'absència de tensió o estrès, tant físic com psicològic (Asociación Parkinson Tenerife sense data). Ens preguntem si, com a professionals de la infermeria, podem ajudar a millorar el control motor dels pacients amb MP mitjançant un tractament no farmacològic $i$, més concretament, amb tècniques de relaxació adequades.

Per tant, l'objectiu d'aquesta revisió de la bibliografia pretén buscar evidència sobre l'efectivitat de les tècniques de relaxació en la millora dels símptomes motors dels malalts de Parkinson.

\section{Mètode}

Es tracta d'una recerca bibliogràfica portada a terme al llarg de març de 2017 . Els descriptors utilitzats per a l'estratègia de recerca van ser en llenguatge controlat i natural «Enfermedad de Parkinson» i «Técnicas de relajación».

Per realitzar la recerca es van utilitzar tres bases de dades: dos pertanyents a fonts secundàries, com són PubMed-Medline i la Biblioteca Virtual de la Salut, i una font terciària, la Biblioteca Cochrane Plus.

Els filtres idiomàtics van ser l'anglès i l'espanyol i el temporal de 2010 a 2017.

\section{Resultats}

Després de la recerca, s'obtingueren un total de $n=101$ articles: $77,23 \%$ en PubmedMedline, 7,92 \% en Pubmed-Clinical Queries, 10,89\% en Biblioteca Cochrane Plus i $3,96 \%$ en BVS; de la mostra resultant es va haver de rebutjar un $88,12 \%(n=89)$ per 
no guardar relació amb l'objectiu de la recerca i un 4,95\% per repetició del mateix article en les diferents bases de dades (figura 1).

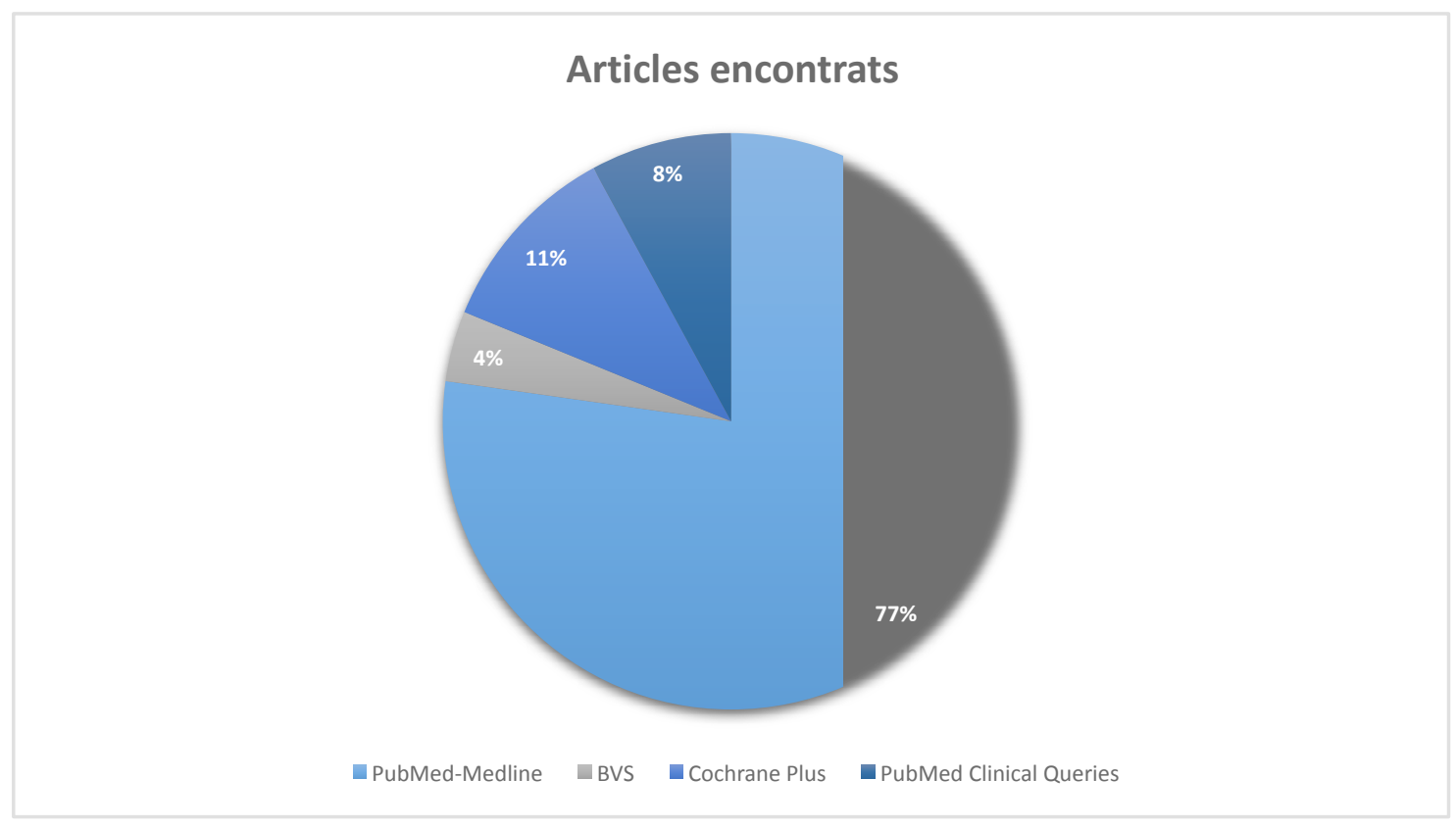

Figura 1. Relació d'articles recuperats en les diferents bases de dades.

En la figura 2 s'observa el nombre d'articles inclosos en el treball distribuïts per any de publicació.

\section{Anys de publicació d'articles}

3

2

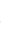

$-$

1

0
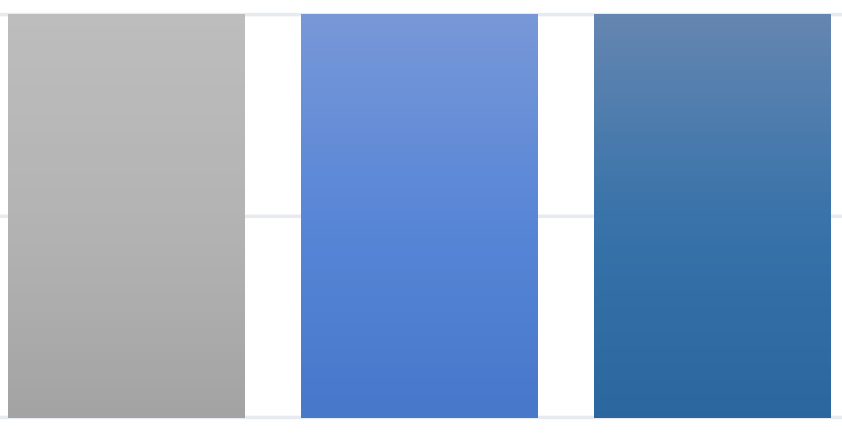

— $2010 \square 2011 \quad 2014 \quad 2015$

Figura 2. Distribució del nombre d'articles encontrats per anys de publicació. 


\section{Discussió i conclusions}

Els resultats d'aquesta recerca han estat estudis que pretenen avaluar l'eficàcia de les tècniques de relaxació en relació amb els símptomes motors de les persones amb la malaltia de Parkinson utilitzant diferents activitats i exercicis de relaxació.

Els símptomes motors en aquests tipus de malalts són una de les principals causes d'incapacitació i es poden exasperar amb l'aparició d'estrès. Tot i que el tractament farmacològic resulta òptim en els primers estadis, dóna lloc a complicacions secundàries que, junt amb la simptomatologia, limiten la seua qualitat de vida i porta els pacients cap a un estil de vida sedentari (Schlesinger, Benyakov, Arik, Suraiya i Schiller 2009; Schlesinger, Benyakov, Erikh i Nassar 2014; van der Kolk, Overeem, de Vries, Kessels, Donders, Brouwer et al. 2015).

Les tècniques de relaxació poden ser un complement important, actuant com a coadjuvant en la reducció de la simptomatologia motora associada a la MP (Ajimsha, Majeed, Chinnavan i Thulasyammal 2014). Al mateix temps també s'ha explorat l'exercici com una estratègia per reduir les complicacions secundàries, ja que proporciona benefici a nivell general de la salut del malalt i un alleujament simptomàtic (van der Kolk, Overeem, de Vries, Kessels, Donders, Brouwer et al. 2015).

Per una banda, es troba l'entrenament autogen. Es tracta d'una tècnica de relaxació que es basa en la visualització guiada d'imatges per relaxar cos i ment (Ajimsha, Majeed, Chinnavan i Thulasyammal 2014); és una de les tècniques que més es fa servir per estudiar el seus efectes sobre els símptomes motors de la MP, més concretament el tremolor (Schlesinger, Benyakov, Arik, Suraiya i Schiller 2009; Schlesinger, Benyakov, Erikh i Nassar 2014; Ajimsha, Majeed, Chinnavan i Thulasyammal 2014; Grupo de Estudio de los Trastornos del Movimiento de la Sociedad Española de Neurología 2017). Aquesta tècnica es compara amb altres exercicis com la música relaxant, com ho fa Schlesinger (Schlesinger, Benyakov, Arik, Suraiya i Schiller 2009; Schlesinger, Benyakov, Erikh i Nassar 2014); però no tots la fan servir de la mateixa forma. Braun i Ajimsha l'empren junt amb la fisioteràpia (Ajimsha, Majeed, Chinnavan i Thulasyammal 2014; Braun, Beurskens, Kleynen, Schols i Wade 2011) i Schlesinger estudia la tècnica en solitari (Schlesinger, Benyakov, Arik, Suraiya i Schiller 2009; Schlesinger, Benyakov, Erikh i Nassar 2014).

Per altra banda, hi ha altres publicacions que també tenen com a objectiu la millora dels símptomes motors, però no utilitzen les tècniques de relaxació per a aconseguirho (van der Kolk, Overeem, de Vries, Kessels, Donders, Brouwer et al. 2015; Reuter, Mehnert, Leone, Kaps, Oechsner i Engelhardt 2011; Loureiro, Bauer i Laureiro 2015). Loureiro aplica en el grup d'intervenció el mètode Watsu de fisioteràpia aquàtica, que es caracteritza per estiraments, moviments de rotació, traccions i manipulacions conjuntes, i el compara amb l'ús únic de la fisioteràpia convencional. Aquest mètode pretén afavorir la relaxació muscular, la millora de la mobilitat i l'estabilitat postural (Loureiro, Bauer i Laureiro 2015).

Amb la mateixa premissa, Reuter estudia tres grups de pacients i compara un programa de caminar, un de marxa nòrdica (NO) i un altre de flexibilitat i relaxació per evidenciar quin disminueix la discapacitat i millora la qualitat de vida (Reuter, Mehnert, Leone, Kaps, Oechsner i Engelhardt 2011).

Per últim, van der Kolk estudia l'efectivitat de l'exercici aeròbic amb elements de joc (exegarming) en un grup control i el compara amb el no aeròbic (estiraments, flexibilitat i exercicis de relaxació), tots dos en un grups de pacients sedentaris, per proporcionar 
un alleujament simptomàtic en la MP (van der Kolk, Overeem, de Vries, Kessels, Donders, Brouwer et al. 2015).

La Unified Parkinson Disease Rating Scale (UPDRS) es una escala validada que s'utilitza per a la investigació i la valoració clínica de la MP que ha demostrat ser sensible als canvis en els estadis i diferències clíniques importants de la malaltia (Schlesinger, Benyakov, Erikh i Nassar 2014). Es troba dividida en quatre blocs diferenciats que mesuren directament els símptomes relacionats amb la discapacitat $i$ la qualitat de vida del pacient: a) estat mental, comportament i estat d'ànim, b) AVD, c) exploració d'aspectes motors, $d$ ) complicacions del tractament (Schlesinger, Benyakov, Erikh i Nassar 2014; Grupo de Estudio de los Trastornos del movimiento de la Sociedad Española de Neurología 2017). En el primers estadis de la MP la progressió clínica és més ràpida i augmenta la puntuació motora de la UPDRS, per la qual cosa es crea la necessitat de buscar tractaments que ajuden a reduir aquesta puntuació motora $\mathrm{i}$, al mateix temps, la taxa de discapacitat dels pacients (Schlesinger, Benyakov, Erikh i Nassar 2014). Ajimsha, Schlesinger, van der Kolk i Reuter en els seus estudis empren aquesta ferramenta per avaluar l'eficàcia de les tècniques aplicades als participants (Schlesinger, Benyakov, Erikh i Nassar 2014; van der Kolk, Overeem, de Vries, Kessels, Donders, Brouwer et al. 2015; Reuter, Mehnert, Leone, Kaps, Oechsner i Engelhardt 2011).

Altres instruments de valoració que s'utilitzen en els diferents estudis són: l'escala de mobilitat del tronc (TMS), l'escala d'equilibri de Berg, la prova i hora Go (Loureiro, Bauer i Laureiro 2015) i el PDQ-39 que valora la salut en relació amb la qualitat de vida (van der Kolk, Overeem, de Vries, Kessels, Donders, Brouwer et al. 2015; Reuter, Mehnert, Leone, Kaps, Oechsner i Engelhardt 2011).

L'estudi de van der Kolk combina diferents proves i qüestionaris per dur a terme la valoració dels participants entre les quals, a més del UPDRS i el PDQ-39, destaquen com a més específiques de la MP SCOPA-sueño i SCOPA-AUT que avaluen l'apartat dels símptomes no motors de la seua investigació (van der Kolk, Overeem, de Vries, Kessels, Donders, Brouwer et al. 2015).

La freqüència de les sessions dels estudis varia entre 2 (Loureiro, Bauer i Laureiro 2015) i 3 (Schlesinger, Benyakov, Arik, Suraiya i Schiller 2009; Schlesinger, Benyakov, Erikh i Nassar 2014; van der Kolk, Overeem, de Vries, Kessels, Donders, Brouwer et al. 2015) a la setmana i altres de 40 sessions al llarg d'un període de més de 8 setmanes (Ajimsha, Majeed, Chinnavan i Thulasyammal 2014). La durada dels estudis ha estat compresa entre 3 mesos (Schlesinger, Benyakov, Arik, Suraiya i Schiller 2009; Schlesinger, Benyakov, Erikh i Nassar 2014) i 6 mesos (van der Kolk, Overeem, de Vries, Kessels, Donders, Brouwer et al. 2015; Reuter, Mehnert, Leone, Kaps, Oechsner i Engelhardt 2011).

Com a limitacions del estudis, cal destacar, que molts d'ells estan formats per una mostra reduïda de pacients i el fet de ser estudis de simple cec (Ajimsha, Majeed, Chinnavan i Thulasyammal 2014).

La majoria d'estudis evidencien que la relaxació a través de la visualització guiada d'imatges dóna resultats significatius per a la millora de símptomes motors i pot ser un possible aliat del tractament farmacològic habitual de la MP quan es compara amb la fisioteràpia convencional o amb la teràpia de música relaxant, tant si s'utilitza sola com si es combina amb la fisioteràpia (Schlesinger, Benyakov, Arik, Suraiya i Schiller 2009; Schlesinger, Benyakov, Erikh i Nassar 2014; Ajimsha, Majeed, Chinnavan i Thulasyammal 2014; Braun, Beurskens, Kleynen, Schols i Wade 2011). 
Però, per una altra banda, quan la relaxació (combinada amb flexibilitat o els estiraments) es compara amb l'exercici aeròbic exegarming, el caminar, el $\mathrm{NO}$ i el mètode Watsu, els resultats ja no són tan favorables per a les tècniques de relaxació (van der Kolk, Overeem, de Vries, Kessels, Donders, Brouwer et al. 2015; Reuter, Mehnert, Leone, Kaps, Oechsner i Engelhardt, 2011; Loureiro, Bauer i Laureiro 2015); aquests tipus d'exercicis físics han evidenciat que són més efectius per al tractament dels símptomes motors perquè proporcionen un alleujament de la simptomatologia del Parkinson (van der Kolk, Overeem, de Vries, Kessels, Donders, Brouwer et al. 2015), una relaxació muscular amb major mobilitat que afavoreix l'estabilitat postural (Loureiro, Bauer i Laureiro 2015) i una reducció del dolor, una millora de l'equilibri i de la salut de qualitat de vida amb una millora de la capacitat d'exercici i de la marxa (Reuter, Mehnert, Leone, Kaps, Oechsner i Engelhardt 2011).

La majoria de les publicacions se centren en intentar millorar la simptomatologia motora dels malalts de Parkinson, ja que, amb l'evolució de la malaltia, aquests poden arribar a incapacitar-los en les seues AVD. L'objectiu general dels diversos estudis encontrats és intentar complementar el tractament farmacològic de la MP amb el no farmacològic (tècniques de relaxació i exercici físic).

En els treballs recuperats s'estudien les tècniques de relaxació, ja siguen aquestes l'objecte de l'estudi o la intervenció amb la qual es comparen altres activitats per avaluar-ne l'efectivitat.

Quan es comparen les tècniques de relaxació amb la música relaxant o la fisioteràpia, apareixen resultats significatius de l'eficàcia d'aquestes tècniques. Però quan l'objecte de la investigació són l'exercici físic aeròbic, el caminar o la NO i es comparen amb altres activitats que inclouen la relaxació, els resultats són desfavorables per a aquestes últimes, ja que l'exercici físic produeix el mateix efecte en els símptomes motors que la relaxació i, a més, millora la marxa i la mobilitat del pacient.

Per tot açò, aquestes activitats podrien arribar a ser un bon aliat del tractament farmacològic per reduir els símptomes del Parkinson i millorar la qualitat de vida dels pacients.

\section{Referències bibliogràfiques}

Ajimsha, M. Sharafudeen, Nisar A. Majeed, Elanchezhian Chinnavan i Ramiah Pillai Thulasyammal. 2014. «Effectiveness of Autogenic Training in improving motor performances in Parkinson's disease». Complementary Therapies in Medicine 22(3): 419-425. http://dx.doi.org/10.1016/j.ctim.2014.03.013

Asociación Parkinson Tenerife [Internet]. Sense data. Santa Cruz de Tenerife: Asociación de Familiares y enfermos de Parkinson de Tenerife. Disponible en: http://www.parkinsontenerife.org/ [Consultat el 5 de febrer de 2017].

Braun, Susy M., Anna J. Beurskens, Melanie Kleynen, Jos M. G. A. Schols i Derick T. Wade. 2011. «Rehabilitation with mental practice has similar effects on mobility as rehabilitation with relaxation in people with Parkinson's disease: a multicentre randomised trial». Journal of Physiotherapy 57(1): 27-34.

Federación Española de Parkinson [Internet]. Sense data. ¿Qué es la enfermedad de Parkinson? Disponible en: http://www.esparkinson.es/espacioparkinson/conocer-la-enfermedad/ [Consultat el 5 de febrer de 2017].

Grupo de Estudio de los Trastornos del Movimiento [Internet]. 2017. Escalas de valoración de la Enfermedad de Parkinson. Disponible en: http://getm.sen.es/profesionales/escalas-de-valoracion/26-getm/escalas-de- 
valoracion/88-unified-parkinson-s-disease-rating-scale-updrs [Consultat el 5 d'agost de 2017].

Loureiro, Ana Paula Cunha, Vladimir Bauer i Claudia da Cunha Loureiro. 2015. «Improving of balance in patients affected by Parkinson's disease by decreasing rigidity». En $19^{\text {th }}$ International Congress of Parkinson's Disease and Movement Disorders. San Diego, California.

Luquín Pulido, María Rosario [Internet]. 2012. Módulo 0: Enfermedad de Parkinson, epidemiología, etiología y manifestaciones clínicas. Neurodidacta. Disponible en: http://www.neurodidacta.es/cursos/guruPrograms/5-enfermedad-deparkinson-y-otros-trastornos-del-movimiento/26-modulo-0-enfermedad-deparkinson-epidemiologia-etiologia-y-manifestaciones-clinicas [Consultat el 5 d'agost de 2019].

Nausieda, Paul, Glorida Bock i Glenna A. Dowling. 2002. Enfermedad de Parkinson: lo que usted y su familia deben saber [Internet]. Florida: National Parkinson Foundation

en:

http://www3.parkinson.org/site/DocServer/Lo_Que_Usted_y_Su_Familia_Debe n_Saber.pdf;jsessionid $=00000000$. app328a?docID $=200 \& \_g a=1.91906458 .120$ 1617.1477065781\&NONCE_TOKEN=11D2FD402B56ED179B91DCB9A127DA AB [Consultat el 5 d'agost de 2019].

Reuter, Iris, Sabine Mehnert, Paola Leone, Manfred Kaps, Matthias Oechsner i Monika Engelhardt. 2011. "Effects of a flexibility and relaxation programme, walking, and nordic walking on parkinson's disease». Journal of aging research. http://dx.doi.org/10.4061/2011/232473

Schlesinger, Ilana, Orna Benyakov, Ilana Erikh, Suheir Suraiya i Yitzhak Schiller. 2009. "Parkinson's disease tremor is diminished with relaxation guided imagery". Movement Disorders 24(14): 2059-2062. http://dx.doi.org/10.1002/mds.22671

Schlesinger, Ilana, Orna Benyakov, Ilana Erikh i María Nassar. 2014. «Relaxation Guided Imagery Reduces Motor Fluctiations in Parkinson's disease». Journal of Parkinson's Disease 4(3): 431-436. http://dx.doi.org/10.3233/JPD-130338

van der Kolk Nocolien M., Sebastiaan Overeem, Nienke M. de Vries, Roy P. C. Kessels, Rogier A. R. T. Donders, Marc A. Brouwer, Daniela Berg, Bart Post i Zoltán Bochdanovits. 2015. "Design of the Park-in-Shape study: a phase II double blind randomized controlled trial evaluating the effects of exercise on motor and non-motor symptoms in Parkinson's disease». BMC Neurology 15. Disponible en: http://bmcneurol.biomedcentral.com/articles/10.1186/s12883015-0312-6 [Consultat el 5 de agosto de 2019]. 